\title{
7 Anstelle eines Fazits: Perspektiven für eine bildungsdidaktische Forschung
}

Bildungsdidaktik lässt sich wie Bildung selbst als Prozess begreifen. Lehrende wie Lernende können selbst einen forschend-evaluativen Blick auf die didaktische Struktur des Lehr-/Lerndesigns einnehmen und über die Wirkungen des didaktischen Settings forschen. So lassen sich Interaktionsprozesse rekonstruieren, die im Lehr-/Lerngeschehen zu Bildungsdynamiken führen können. Durch diese Rekonstruktion wird es möglich, Kausalbedingungen zu identifizieren, die das Entstehen eines Bildungsraums fördern:

- Welche Konstellationen in einem Interaktionsgeschehen können Bildungsdynamiken und Bildungslernen hervorrufen? (es ließe sich beispielsweise danach fragen, welche Bedingungen erfüllt sein müssen, damit sich beispielsweise Bildungslernen im Zuge von Gruppenarbeiten entfalten kann).

Die Beforschung von Lernräumen und die evaluative Prüfung, ob es sich hierbei um Bildungsräume handelt, rückt die integrative Bildungsforschung in die Nähe der Evaluationsforschung (vgl. Kergel \& Heidkamp 2015, 2018; Kergel 2018). Die Nähe der Beforschung von Bildungsräumen zur Evaluation von Lehr-/Lerngeschehen ergibt sich aus der theoretisch fundierten, normativen Dimension von Bildung: Besteht der Anspruch, einen Bildungsraum im Zuge pädagogischer Praxis zu realisieren, lässt sich das Vorhandensein normativer Aspekte von Bildung als ein Sollwert bestimmen. Die normative Dimension von Bildung kann dann als Qualitätsmerkmal für pädagogische Interaktionsprozesse verstanden werden, wenn in Bezug auf diese pädagogischen Interaktionsprozesse der Anspruch besteht, Bildung bzw. einen Bildungsraum zu realisieren. 